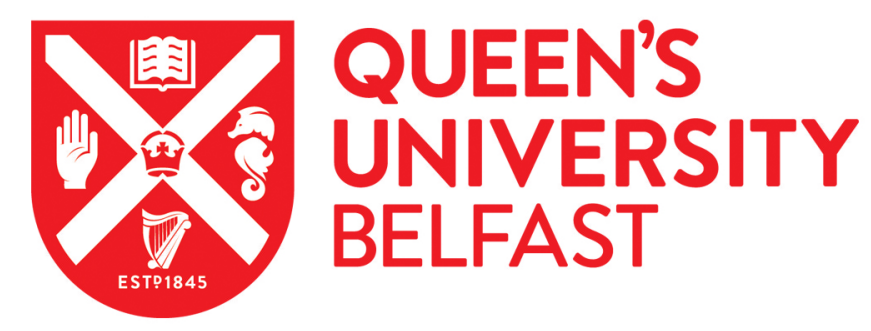

\title{
Phylogenetic analysis of porcine reproductive and respiratory syndrome virus isolates from Northern Ireland
}

Smith, N., Power, U. F., \& McKillen, J. (2018). Phylogenetic analysis of porcine reproductive and respiratory syndrome virus isolates from Northern Ireland. Archives of Virology. https://doi.org/10.1007/s00705-018-3886-7

Published in:
Archives of Virology

Document Version:

Peer reviewed version

Queen's University Belfast - Research Portal:

Link to publication record in Queen's University Belfast Research Portal

Publisher rights

(c) 2018 Springer-Verlag GmbH Austria, part of Springer Nature. This work is made available online in accordance with the publisher's policies. Please refer to any applicable terms of use of the publisher.

\section{General rights}

Copyright for the publications made accessible via the Queen's University Belfast Research Portal is retained by the author(s) and / or other copyright owners and it is a condition of accessing these publications that users recognise and abide by the legal requirements associated with these rights.

Take down policy

The Research Portal is Queen's institutional repository that provides access to Queen's research output. Every effort has been made to ensure that content in the Research Portal does not infringe any person's rights, or applicable UK laws. If you discover content in the Research Portal that you believe breaches copyright or violates any law, please contact openaccess@qub.ac.uk. 
Phylogenetic analysis of porcine reproductive and respiratory syndrome virus isolates from Northern Ireland

3

Natalie Smith ${ }^{1,2}$, Ultan F. Power ${ }^{2}$ and John McKillen ${ }^{1}$

${ }^{1}$ Agri-Food and Biosciences Institute, Veterinary Science Division, Stormont, Belfast, BT4 3SD

2 Centre for Experimental Medicine, School of Medicine, Dentistry and Biomedical Sciences, Queen's University Belfast, Medical Biology Centre, Belfast, BT9 7BL

\section{Abstract}

To investigate the genetic diversity of porcine reproductive and respiratory syndrome virus (PRRSV) in Northern Ireland, the ORF5 gene from 9 field isolates was sequenced and phylogenetically analysed. The results revealed relatively high diversity amongst isolates with 87.6-92.2\% identity between farms at the nucleotide level and 84.1-93.5\% identity at the protein level. Phylogenetic analysis confirmed that all 9 isolates belonged to the European (type 1) genotype, and formed a cluster within the subtype 1 subgroup. This study provides the first report on PRRSV isolate diversity in Northern Ireland.

Porcine reproductive and respiratory syndrome virus (PRRSV), the causative agent of porcine reproductive and respiratory syndrome (PRRS), is a small, enveloped, positive sense, single stranded RNA virus (genus Arterivirus). It is responsible for inducing reproductive failure in sows and respiratory disease in growing pigs [1]. Two genotypes of PRRSV are recognised internationally, European (type 1) and North American (type 2). The type 2 virus was originally isolated in pig herds in America in 1987 [2], while type 1 was first identified in European pigs in 1991 [3]. Although the first outbreaks occurred close together, the two genotypes have high sequence divergence and share only 50-70\% genetic identity [4-7]. There are at least 4 subtypes of type 1 PRRSV, with different worldwide and European distributions, as well as variations in pathogenicity [8]. Similarly, type 2 PRRSV is further divided into several lineages [9].

The high strain diversity of PRRSV is consistent with other RNA viruses, and results in rapidly emerging virus variants that lead to recurrent disease outbreaks and increased difficulty with virus control [10-12]. As the island of Ireland is geographically isolated we hypothesised that Northern Ireland (NI) may have circulating strains of virus that are different from those found in Great Britain and the rest of Europe. The genotype of the virus has obvious implications for diagnostics and vaccination, with only limited protection afforded against heterologous strains. In this study, the ORF5 gene from PRRSV field isolates in NI was sequenced and compared with those from modified live virus (MLV) vaccine sequences and other European isolates. The genetic diversity of PRRSV strains in NI relative to vaccine and European wild type strains was examined and the implications for PRRSV-associated disease management discussed.

Seven lung samples and 2 mesenteric lymph node (MLN) samples were obtained from 9 pigs from 5 farms in NI. Ten percent w/v tissue homogenates were prepared and RNA was extracted using standard methods. A previously described primer set [13] was used to amplify a $780 \mathrm{bp}$ PCR product containing the complete ORF5 gene 
of PRRSV and resultant amplicons were sequenced commercially. Raw sequence reads were analysed and trimmed using Geneious version 6 [14]. Clustal W was used to align the NI nucleotide sequences with a selection of chronologically and geographically varied wildtype PRRSV type 1 isolates from subtypes 1-4 as well as MLV sequences from GenBank. Phylogenetic trees were generated using the neighbour-joining method [15] in MEGA 6 [16] with 1000 iterations for bootstrapping. Predicted protein sequences were also aligned using Clustal W. The sequences of the predicted GP5 proteins were searched for motifs associated with Nlinked glycosylation [17] and a previously described neutralising epitope [18, 19].

All field isolates from NI were analysed and confirmed as type 1, subtype 1 PRRSV isolates by sequencing and BLASTn analysis. Comparison of aligned sequences showed the expected high degree of variability among sequences (Table 1). Analysis of nucleotide sequences from NI isolates showed $87.62-92.2 \%$ similarity between farms. Within farms there was a high level of sequence conservation (99.5-100\%).

Nucleotide sequence homology between NI strains and selected European sequences ranged from $76.2 \%$ (subtype 2) to $92.7 \%$ (subtype 1). The NI strains were all subtype 1 and had between $82.5 \%$ and $92.7 \%$ similarity with the other sequences in this subtype. Nucleotide similarity was lower between the NI sequences and other European subtypes (76.2-80\% with subtype 2, 79.2-83.5\% with subtype 3 and 77.6 $82.1 \%$ with subtype 4 ). NI sequences were also compared to the ORF5 sequences of 4 commercially available PRRSV MLV vaccines. Sequence comparison showed nucleotide homology ranging from 84.7-92.9\%. The majority of NI PRRSV isolates were collected in 2015 ( 8 sequences from 4 farms) and these clustered together as a distinct subtype 1 subgroup on the phylogenetic tree as part of a larger subgroup (Figure 1). One sequence from farm 13320-12 collected in 2012 clustered with UK and North American strains, most closely clustering with EuroPRSSV (Accession no. AY366525) [20].

The predicted proteins were 201 amino acids in length for all NI sequences, with homology ranging from $74.1-100 \%$ with the other international sequences (Table 1), reflecting the differences evident at the nucleotide level. NI PRRSV protein sequences were $81.6-91.5 \%$ identical to vaccine strains.

The presence of a neutralisation epitope located in the N-terminus of the GP5 ectodomain was observed between residues 29-35 (WSFADGN) (Fig. 2), as previously described [19]. The MLV vaccine-derived sequences and 3 other subtype 1 sequences had a slightly different motif of WSFVDGN. Interestingly, the $2 \mathrm{NI}$ sequences from farm 1776-15 had differences in the neutralisation epitope at residues 30 and 35, resulting in a motif of WPFADG A. All ORF5 sequences displayed 3 potential N-linked glycosylation sites, at residues 37-39 (Asn-Ser-Ser), 46-48 (AsnLeu-Ser) and 53-55 (Asn-Gly-Thr). One NI sequence from farm 5612-15 displayed an additional N-linked glycosylation site at residues 38-40 (Asn-Ser-Thr), which overlapped with the sequon at residues 37-39 to contain the sequence Asn-Asn-SerThr.

ORF5 was targeted for phylogenetic analysis as it encodes the most variable structural protein, GP5 $[13,21]$. GP5 is also the major target for virus neutralising antibodies [22] and, as such, is important in relation to protection derived from previous infection or vaccination. Alignment of the complete ORF5 sequences revealed 
nucleotide homology ranging from $87.6-100 \%$ between NI field strains. The difference in homology between these virus strains is consistent with the genetic diversity previously reported for PRRSV isolates in the United Kingdom [23], Italy [13], Denmark [24], the United States [6], China [25] and Poland [26]. Phylogenetic analyses confirmed that all NI PRRSV isolates are of the European genotype, and placed the 2015 NI sequences into a distinct cluster within this group (Fig. 1). As expected, virus sequences from the same farms were closely related.

101

102

103

104

105

106

107

108

109

110

111

112

113

114

115

116

117

118

119

120

121

122

123

124

125

126

127

128

129

130

131

132

133

134

135

136

137

138

139

140

Interestingly, sequence diversity was observed between the ORF5 nucleotide and predicted protein sequences of circulating NI isolates and those of MLV vaccine sequences. MLV vaccines are capable of reducing the clinical signs associated with PRRSV infection, as well as viremia and viral shedding [27]. However, the efficacy of commercially available PRRSV type 1 MLV vaccines is variable and is characterised by a delayed neutralising antibody response [28]. Importantly, the genetic diversity of circulating strains may result in diminished protection afforded by the vaccines. For example, in vaccine efficacy studies, vaccination of pigs with MLV vaccine resulted in only partial protection against challenge with a heterologous East European PRRSV type 1 subtype 3 strain (Lena strain) [29]. The ORF5 of the Lena sequence was found to be $88 \%$ identical to the MLV vaccine at the protein level. The levels of amino acid homology between NI isolates was as low as $81.6 \%$ (farm 13320-12). It is not known what level of protection would be provided by MLV vaccines against this Northern Irish field isolate. However, such large differences may have a significant impact on vaccine efficacy. Consequently, continued monitoring of local PRRSV sequence variation is necessary. Nonetheless, vaccination against PRRSV with MLV vaccines remains one of the most important tools for control of the virus.

Changes in neutralising epitopes were shown to alter the effectiveness of neutralising antibodies [30]. A number of studies have described neutralisation epitopes in type 2 PRRSV [18, 31]. While the putative neutralising epitopes have not been as well documented for type 1 PRRSV we explored one epitope situated between residues 2935 [19, 22]. The NI isolates from farm 1776-15 had mutations in amino acids in this neutralisation epitope compared to the majority of subtype 1 strains and the vaccine sequences. This suggests that vaccine efficacy may be compromised in NI. However, further studies are evidently required to determine the significance of these amino acid changes on vaccine efficacy.

As well as resulting in variation in neutralising epitopes, genetic variation can lead to changes in N-linked glycosylation sites and this can have an effect on the recognition of the neutralisation epitope [32]. Three potential N-linked glycosylation sites were identified in the GP5 ectodomain of NI PRRSV strains at residues 37-39, 46-48 and 53-55. This is consistent with Pesente et al. [13] and Frossard et al. [23], who identified the same predicted glycosylation sites on the GP5 protein of Italian and British PRRSV isolates, respectively. Interestingly, in one NI PRRSV strain an additional N-linked glycosylation site that overlapped with the sequon present at residue 37 was identified. Glycosylation of the viral envelope protein is a mechanism for immune evasion and several studies demonstrated a role for PRRSV GP5 glycosylation modification in evading host immune responses [33, 34]. Indeed, removal of N-glycosylation sites surrounding the neutralisation epitope of PRRSV GP5 resulted in increased sensitivity to neutralising antibodies [33] and convalescent- 
phase serum [17]. Consistent with the NI strains, the majority of subtype 1 sequences studied had predicted N-glycosylation sites at residues 37-39, 46-48 and 53-55. Importantly, all but one of the MLV vaccine strains (Porcilis) had no predicted Nglycosylation at residues 37-39, suggesting that the neutralising epitope may be more immunogenic for these vaccine strains. The presence of $\mathrm{N}$-glycosylation at this site in the NI field isolates could compromise the immunity provided by the vaccines.

In conclusion, despite the genetic diversity observed between NI PRRSV isolates, these strains mostly clustered together in a distinct group within the European genotype. These data demonstrated relatively high genetic variability among PRRSV strains in NI and this variability poses significant challenges to the control of PRRS through vaccination. The geographical isolation of the island of Ireland may be a positive factor in terms of prevention of the introduction of diverse strains of PRRSV. However, the diversity between Northern Irish PRRSV strains evident in this study suggests that a more in depth surveillance on an all-island basis will be important in understanding, and locally controlling, PRRSV disease in Ireland.

\section{Acknowledgements}

The authors would like to thank the Department of Agriculture, Environment and Rural Affairs (DAERA) for funding this study.

\section{Funding}

This study was part of a PhD studentship funded by the Department of Agriculture, Environment and Rural Affairs (DAERA) for Northern Ireland.

\section{Conflict of Interest}

All the authors declare that they have no conflict of interests.

\section{Ethical approval}

This article does not contain any studies with human participants or animals performed by any of the authors.

\section{References}

1. Rossow KD (1998) Veterinary Pathology Online. Vet Pathol 35:1-20.

2. Keffaber K. (1989) Reproductive failure of unknown etiology. Am Assoc Swine Pr Newsl 1-10.

3. Wensvoort G, Terpstra C, Pol JM, et al (1991) Mystery swine disease in The Netherlands: the isolation of Lelystad virus. Vet Q 13:121-130.

4. Allende R, Lewis TL, Lu Z, et al (1999) North American and European porcine reproductive and respiratory syndrome viruses differ in non-structural protein coding regions. Gen Virol 80:307-15

5. Meng X. (2000) Heterogeneity of porcine reproductive and respiratory syndrome virus: implications for current vaccine efficacy and future vaccine development. Vet Microbiol 74:309-329.

6. Fang Y, Schneider P, Zhang WP, et al (2007) Diversity and evolution of a newly emerged North American Type 1 porcine arterivirus: Analysis of isolates collected between 1999 and 2004. Arch Virol 152:1009-1017. 
183 7. Dokland T (2010) The structural biology of PRRSV. Virus Res 154:86-97.

184 8. Stadejek T, Stankevicius A, Murtaugh MP, Oleksiewicz MB (2013) Molecular evolution of PRRSV in Europe: Current state of play. Vet Microbiol 165:2128.

9. Brar MS, Shi M, Murtaugh MP, Leung FCC (2015) Evolutionary diversification of type 2 porcine reproductive and respiratory syndrome virus. $\mathrm{J}$ Gen Virol 96:1570-1580.

10. Marozin S, Gregory V, Cameron K, et al (2002) Antigenic and genetic diversity among swine influenza viruses in Europe. J Gen Virol 83:735-745.

11. Midgley SE, Banyai K, Buesa J, et al (2012) Diversity and zoonotic potential of rotaviruses in swine and cattle across Europe. Vet Microbiol 156:238-245.

12. Gunn L, Collins PJ, O'Connell MJ, O'Shea H (2015) Phylogenetic investigation of enteric bovine coronavirus in Ireland reveals partitioning between European and global strains. Ir Vet J 68:31.

13. Pesente P, Rebonato V, Sandri G, et al (2006) Phylogenetic analysis of ORF5 and ORF7 sequences of porcine reproductive and respiratory syndrome virus (PRRSV) from PRRS-positive Italian farms: a showcase for PRRSV epidemiology and its consequences on farm management. Vet Microbiol 114:214-24.

14. Kearse M, Moir R, Wilson A, et al (2012) Geneious Basic: An integrated and extendable desktop software platform for the organization and analysis of sequence data. Bioinformatics 28:1647-1649.

15. Saitou N, Nei M (1987) The neighbour-joining method: a new method for reconstructing phylogenetic trees. Mol Biol Evol 4:406-425.

16. Tamura K, Stecher G, Peterson D, et al (2013) MEGA6: Molecular evolutionary genetics analysis version 6.0. Mol Biol Evol 30:2725-2729.

17. Wei ZZ, Lin T, Sun LC, et al (2012) N-Linked Glycosylation of GP5 of Porcine Reproductive and Respiratory Syndrome Virus Is Critically Important for Virus Replication In Vivo. J Virol 86:9941-9951.

18. Ostrowski M, Galeota J a, Jar a M, et al (2002) Identification of Neutralizing and Nonneutralizing Epitopes in the Porcine Reproductive and Respiratory Syndrome Virus GP5 Ectodomain Identification of Neutralizing and Nonneutralizing Epitopes in the Porcine Reproductive and Respiratory Syndrome Virus GP. J Virol 76:4241-4250.

19. Wissink EHJ, van Wijk H a R, Kroese M V., et al (2003) The major envelope protein, GP5, of a European porcine reproductive and respiratory syndrome virus contains a neutralization epitope in its $\mathrm{N}$-terminal ectodomain. J Gen Virol 84:1535-1543.

20. Ropp SL, Wees CEM, Fang Y, et al (2004) Characterization of emerging European-like porcine reproductive and respiratory syndrome virus isolates in the United States. J Virol 78:3684-3703.

21. Meng XJ, Paul PS, Halbur PG, Morozov I (1995) Sequence comparison of open reading frames 2 to 5 of low and high virulence United States isolates of porcine reproductive and respiratory syndrome virus. J Gen Virol 76:31813188.

22. Weiland E, Wieczorek-Krohmer M, Kohl D, et al (1999) Monoclonal antibodies to the GP5 of porcine reproductive and respiratory syndrome virus are more effective in virus neutralization than monoclonal antibodies to the GP4. Vet Microbiol 66:171-186.

23. Frossard J-P, Hughes GJ, Westcott DG, et al (2013) Porcine reproductive and 
respiratory syndrome virus: genetic diversity of recent British isolates. Vet Microbiol 162:507-18.

24. Kvisgaard LK, Hjulsager CK, Kristensen CS, et al (2013) Genetic and antigenic characterization of complete genomes of Type 1 Porcine Reproductive and Respiratory Syndrome viruses (PRRSV) isolated in Denmark over a period of 10 years. Virus Res 178:197-205.

25. Li Y, Wang X, Jiang P, et al (2009) Genetic variation analysis of porcine reproductive and respiratory syndrome virus isolated in China from 2002 to 2007 based on ORF5. Vet Microbiol 138:150-155.

26. Stadejek T, Oleksiewicz MB, Scherbakov A V., et al (2008) Definition of subtypes in the European genotype of porcine reproductive and respiratory syndrome virus: Nucleocapsid characteristics and geographical distribution in Europe. Arch Virol 153:1479-1488.

27. Renukaradhya GJ, Meng X-J, Calvert JG, et al (2015) Live porcine reproductive and respiratory syndrome virus vaccines: Current status and future direction. Vaccine 33:4069-4080.

28. Charerntantanakul W (2012) Porcine reproductive and respiratory syndrome virus vaccines: Immunogenicity, efficacy and safety aspects. World J Virol $1: 23$.

29. Trus I, Bonckaert C, van der Meulen K, Nauwynck HJ (2014) Efficacy of an attenuated European subtype 1 porcine reproductive and respiratory syndrome virus (PRRSV) vaccine in pigs upon challenge with the East European subtype 3 PRRSV strain Lena. Vaccine 32:2995-3003.

30. Kim W Il, Yoon KJ (2008) Molecular assessment of the role of envelopeassociated structural proteins in cross neutralization among different PRRS viruses. Virus Genes 37:380-391.

31. Plagemann PGW, Rowland RRR, Faaberg KS (2002) The primary neutralization epitope of porcine respiratory and reproductive syndrome virus strain VR-2332 is located in the middle of the GP5 ectodomain. Arch Virol 147:2327-2347.

32. Faaberg KS, Hocker JD, Erdman MM, et al (2006) Neutralizing antibody responses of pigs infected with natural GP5 N-glycan mutants of porcine reproductive and respiratory syndrome virus. Viral Immunol 19:294-304.

33. Ansari IH, Kwon B, Osorio F a, Pattnaik AK (2006) Influence of N-Linked Glycosylation of Porcine Reproductive and Respiratory Syndrome Virus GP5 on Virus Infectivity, Antigenicity, and Ability To Induce Neutralizing Antibodies. J Virol 80:3994-4004.

34. $\mathrm{Vu}$ HLX, Kwon B, Yoon K-J, et al (2011) Immune evasion of porcine reproductive and respiratory syndrome virus through glycan shielding involves both glycoprotein 5 as well as glycoprotein 3. J Virol 85:5555-64.

\section{Legends}

Fig. 1 Neighbour-joining phylogenetic tree based on complete PRRSV ORF5 nucleotide sequences. Scale bar indicates an evolutionary distance of 10.0 nucleotides per position in the sequences. Sequences from the Northern Irish strains from this study are in blue, while MLV vaccine sequences are in red. Tree is rooted with VR2332 type 2 PRRSV sequence.

Fig. 2 Alignment of the predicted amino acid sequence of the PRRSV GP5 protein of the Northern Irish strains and selected European and vaccine strains. Three potential 
283 N-linked glycosylation sites are denoted with an arrow $(\downarrow)$ and the neutralisation 284 epitope (WSFADGN) is highlighted by a box. 


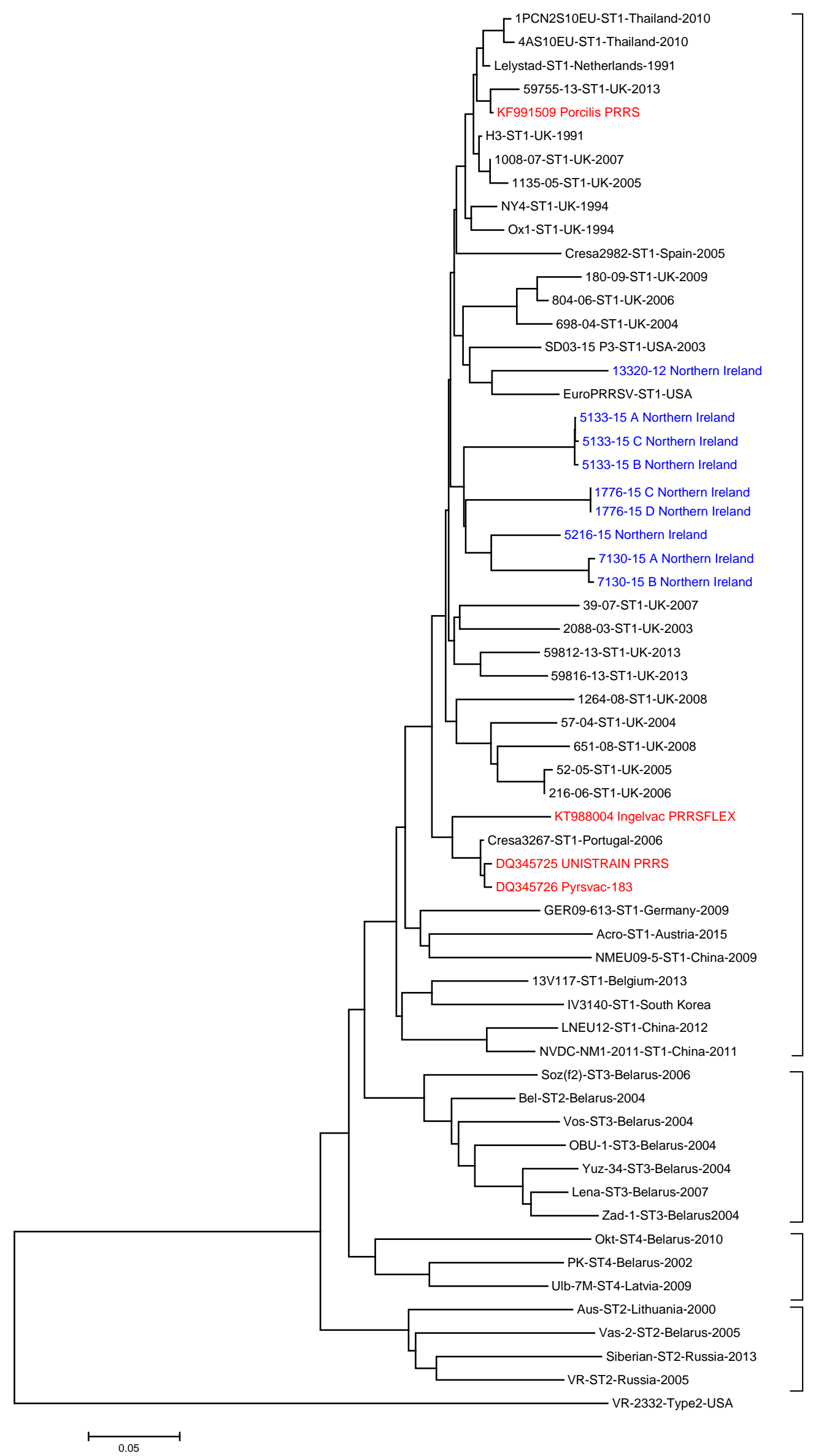

Subtype 1

Subtype 3

Subtype 4

Subtype 2 


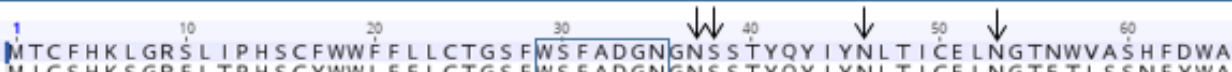
MICSHKSGRFLTPHSCYWWLFFLCTGSFWSFADGNGNSSTYOY IYNLTICE LNGTETLSSNFYWA MICSHKSGRFLTPHSCYWWLFFLCTGSFWSFADGNGNSSTYOY IYNLTICE LNGTEALSSNFYWA MTCFHKLGRFLTPHYCFWWLFLLCTGLSWPFADGAONSSTYOY IYNLTICE LNGTDWLSGHF TWA MTCFHKLGRFLTPHYCFWWLFLLCTGLSWPFADGARNSSTYOY IYNLTICE LNGTDWLSGHF TWA MICSHKSGRFLTPHSCYWWLFFLCTGS FWS FADGNGNNS TYOY IYNLT ICE LNGTDWLSGH PDWA
MRCSHKLGRFLTPHSCFWWLFLLCTGLSWWFADGNGNSSTYOS IYNLTICE LNGTEWLSGHFDWA MRCSHKLGRFLTPHSCFWWLFLLCTGLSWSFADGNGNSSTYQS IYNLTI IE LNGTEWLSGHFDWA UKCSHRLGRS WKCSHKL MRCSHKLGRSLIPHSCEWWLELLCIGLHWSEADGNGNSSTYOYIYNLTICELNGTHWLSSHEDWA MRCSYKLGRSLILHSCSWWFFLLCTGLSWSFADGNGNNSTYOYIYNLTI CE LNGTNWLSGHFDWA

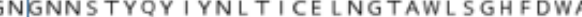
MRCSHKLGRFLTPHSCFWWLFLLCTGLSWSFADGNGDSSTYOY IYNLT ICE LNGTDWLSSHFGWA MRCSHKLGRFLTPHSCFWRLFLLCTGLSWSFADGNGD S STYQY IYNLT I CE LNGTDWLSSHFGWA MRCSHKLGRFLTPHSCFWWLFLLCTGLSWSFADGNGDS STYOY IYNLTI IEE LNGTDWLSSHFGWA MRCSSHKLGRFLTPHSCFWLFLLCIGLSWSFADGNGSS STYOY IYNLT I CE LNGTDWLSSHF GWA URCSHKL LRFLTPHSCFWWFE L CTGLSWSFADGNGNSSTYOYIYNLTICELNGTDWISSHFGWA MRCSHKLGRF TPHSCFWWLFLLCTGLSWSFADGNGNSSTYOYIYNLTICELNGTDWLSSHFGWA MRCSHKLGCFSTTOSCFWWFILLCTGLSWSFADGNGNS STYOYIYNLTICE LNGTGWLSNHFDWA MRCSHKLGRFLTPOSCFWWLFLLCTGLSWSFADGNGDSSTYOY IYNLTICE LNGTDWLSDHFVWA MRCFHKLERFSTPHSCFWLFLLCTGLFWSFADGNGNNSTYOY IYNLTICE LNGTDWLSGRFDWA MRCS HKLLRFLTPHSCFWWLFLLCTGLSWSFADGNGNSSTYQY YYNLTICE LNGTDWLSGHFDWA MICS MRCSSHKSGRFLTPR SCFWWFFFLCTGLSWSFADGNGNSSTYOY IYNLTI CQLNGTDWLSGR FDWA WR MRCSRKSGRF TTPRSCFWWLFELCTGLSSFADGNONS STYOYIYNLTICE LNGTNWLPGHFDWA MRCSHKSGLFLIPHSCFWWLFLLCTGLSWSFADGNGNS STYOYIYNLTICE LNGTDWLSNHFDWA MRCSHKSGR LTPHSCFWWLFLLCTGLSWSFADGNGNSSTYOYIYNLTICE LNGTEWLSSHFDWA MRCSHKLERFLTPHSCFWWLFLLCTGLSWSFADGNGNS SAYOY IYNLTICELNGTEWLSNHFRWA MRCSHKLERFLTPHSCFWWLFLLCTGLSWSFVDGNDSS STYOYIYNLTICE LNGTEWLPSHFDWA MRCSHKLECFLTPHSCFWWLFLLCTGLSWSFVDGNDSSSTYOYIYNLTICE LNGTESLSSHFDWA MRCSHKLERFLTPHSCFWWLFLLCTGLSWSFVDGNDDSSTYOY IYNLTICE LNGTEWLSSHFDWA MKCSCKLGHFLTPHSCFWWLFLLCTGLSWSFVDGNDDSSTSOYIYNLTICE LNGTEWLSGHFDWA MRCS HKLERFLIQRSCLWWLFLLYTGLPWSFVDGNDNSSTYQY I YNLTI IEE LNGTEWLSGHFDWA MRCSHK URCSHKLEHFL MRCSHKLEHFLTPHSCFWWLFLLCTGLSWSEADGNGNS STYOY YNLTICELNGTDWLSEHFYWA MRCSHKSGLFLTPHSCFWLPFLLCTGLSWSFADGNGNSSTYOY YNLTICE LNGTTWLSAHFDWA MRCSYKLGCFLIPHSCFWWLFFLCTGLSWSFANGND I S TYOY IYNLTICELSGTAWLSNHFYWA MRC SHKLARFLTPHSCFWWLFLLCTGLSWSFADGNONSSTYQY IYNMTICE LNGTAWLSNKFYWA MRCSRTLGQPSTHHSYLWWLFLLCTGLSWSFADGNGNS STYLY IYNLTI CE LNGTEWLVNHFDWA MRCSRTLGQP STHHFYLWWLFLLCIGLSWSFADGNGNS STYOY IYDLTI IEE LNGTNWLASRF SWV

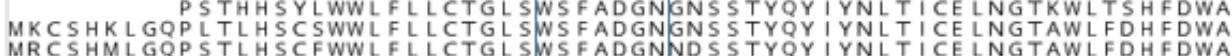
MKCSHKLGOP I HSCFWWLFILCTGLSWSFADGNGNSSTYOY IYNLTICE LNGTAWLSTH FDWA MRCSHRSGOPLTLYSYSWWLFLLCTGLSWSEVDGNGNSSTYOYIYNLTICE LNGTAWLSSHFDWA RSTSSSYFWWLFLLCTGLSWSFADGNGNS STYOY YNLTI PLIPCSYFWWLFLLCTGLSWSFADGSIGSS STYOY IYNLTICE LNGTAWLSYKFHWA P

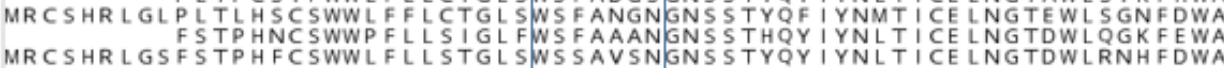
MRCSHRLGSFSTPHFCSWWLFLLSTGLSWSSA S SNGNSSTYOY YNLTICE LNGTDWLRNHFDWA MRCSHKLGSFSTPYFCFWLLSLLSTGLFWS SVASPNGSTYOY IYNLTI IE LNGTDWLSEKFPWV 
Table 1. The minimum and maximum percentage homologies of PRRSV ORF5 nucleotide and protein sequences. Comparisons were within and between Northern Ireland farms, between Northern Ireland sequences and type 1 PRRSV subtypes (1 4) and between Northern Ireland sequences and selected MLV vaccine sequences.

\begin{tabular}{|c|c|c|c|c|}
\hline $\begin{array}{c}\text { ORF5 sequences } \\
\text { compared }\end{array}$ & $\begin{array}{c}\text { Minimum } \\
\text { nucleotide } \\
\text { homology }\end{array}$ & $\begin{array}{c}\text { Maximum } \\
\text { nucleotide } \\
\text { homology }\end{array}$ & $\begin{array}{c}\text { Minimum } \\
\text { protein } \\
\text { homology }\end{array}$ & $\begin{array}{c}\text { Maximum } \\
\text { protein } \\
\text { homology }\end{array}$ \\
\hline $\begin{array}{c}\text { NI - NI (between } \\
\text { farms) }\end{array}$ & 87.6 & 92.2 & 84.1 & 93.5 \\
\hline NI - NI (within farms) & 99.5 & 100 & 99 & 100 \\
\hline NI - Subtype 1 & 82.5 & 92.7 & 82.1 & 92.5 \\
\hline NI - Subtype 2 & 76.2 & 80 & 74.1 & 82.6 \\
\hline NI - Subtype 3 & 79.2 & 83.5 & 75.6 & 86.1 \\
\hline NI - Subtype 4 & 77.6 & 82.1 & 77.6 & 85.3 \\
\hline NI - Porcilis PRRS & 90.3 & 92.9 & 87.6 & 91.5 \\
\hline MLV & 87.3 & 89.8 & 83.6 & 90.1 \\
\hline NI - Pyrsvac-183 & 87.6 & 89.9 & 85.1 & 91 \\
\hline NI - UNISTRAIN & 88.1 & 81.6 & 87.6 \\
\hline $\begin{array}{c}\text { NI - Ingelvac } \\
\text { PRRSFLEX }\end{array}$ & 84.7 & 89.6 & 9 & 9 \\
\hline
\end{tabular}

